\title{
Le rapport au savoir en formation continue : les effets de l'inconscient chez un professeur des écoles. Une étude de cas
}

The non use of knowledge transmitted in swimming training courses in continuing education; the unconscious effects for a primary school teacher. A study case

\section{Matthieu Laugier, Yves Félix Montagne et Denis Loizon}

\section{QpenEdition}

\section{Journals}

Édition électronique

URL : https://journals.openedition.org/ree/709

DOI : 10.4000/ree.709

ISSN : $1954-3077$

Éditeur

Nantes Université

Référence électronique

Matthieu Laugier, Yves Félix Montagne et Denis Loizon, « Le rapport au savoir en formation continue les effets de l'inconscient chez un professeur des écoles. Une étude de cas », Recherches en éducation [En ligne], 38 | 2019, mis en ligne le 01 novembre 2019, consulté le 08 décembre 2022. URL : http:// journals.openedition.org/ree/709; DOI : https://doi.org/10.4000/ree.709

\section{cc) () $\ominus$}

Creative Commons - Attribution - Pas d'Utilisation Commerciale - Pas de Modification 4.0 International - CC BY-NC-ND 4.0

https://creativecommons.org/licenses/by-nc-nd/4.0/ 


\title{
Le rapport au savoir en formation continue : les effets de l'inconscient chez un professeur des écoles. Une étude de cas
}

\author{
Matthieu Laugier, Yves Félix Montagne \& Denis Loizon ${ }^{1}$
}

\begin{abstract}
Résumé
À partir d'une étude de cas, cette recherche interroge les raisons du non-réinvestissement par un professeur des écoles des savoirs transmis en formation continue « natation ». Inscrite dans une perspective clinique de recherche orientée par la psychanalyse, elle explore les manifestations de l'inconscient à l'œuvre dans cette coupure du fil du savoir. La réalisation d'entretiens cliniques de recherche articulés à l'observation filmée d'une séance de natation a permis le recueil des données. L'analyse interprétative de la parole du sujet fait émerger plusieurs causes inconscientes au non-réinvestissement des savoirs transmis en formation, liées aux concepts du «Réel », de « jouissance » et de " déjà-là ». Cette identification d'une structure singulière du rapport au savoir d'un sujet ouvre des perspectives professionnelles visant la construction d'une posture clinique de formateur.
\end{abstract}

En 2017, un rapport relatif à la formation continue des professeurs des écoles et à son évaluation $^{2}$ a mis en avant le caractère indispensable de cette formation mais aussi son manque d'efficacité. Ce diagnostic fait écho à un constat d'un « non appris » dans le cadre d'une formation continue « natation » destinée à des professeurs des écoles. Si certains disent apprécier la qualité de la formation, ils ne montrent cependant pas, quand ils enseignent, un réinvestissement des contenus reçus en formation. Quelle analyse effectuer et quelles perspectives entrevoir pour l'action du formateur quand l'appropriation des savoirs issus de la formation ne se fait pas ou se situe en décalage avec le désir du formateur? Ce constat vient questionner la notion de « rapport au savoir » (Beillerot, 1989). Qu'est-ce qui pourrait expliquer le décalage entre le " transmis » et le "réalisé »? (Loizon, 2005). Quels déterminants inconscients provoqueraient le cas échéant cette dénégation en acte ?

L'objectif de ce travail est d'appréhender les déterminants subjectifs organisant le rapport au savoir de professeurs des écoles engagés dans un processus de transmission/appropriation de savoirs en natation.

Cette étude s'appuiera sur le cadre théorique de la psychanalyse et une méthodologie clinique de recueil et d'interprétation de la parole. Une étude de cas montrera que Gilles ${ }^{3}$, professeur des écoles concerné par l'enseignement de la natation, a de « vraies » raisons pour ne pas pouvoir réinvestir les savoirs transmis en formation. Questionner ce fait engage aussi le responsable de formation à l'analyse des effets de ses interventions.

\section{Cadre théorique}

Le cadre théorique retenu est celui de la psychanalyse, celle des écrits de Sigmund Freud et de l'enseignement de Jacques Lacan, définie comme l'analyse du psychisme à travers le postulat de l'existence de l'inconscient.

\footnotetext{
${ }^{1}$ Matthieu Laugier, doctorant, Institut de recherche sur l'éducation (IREDU), Université de Bourgogne. Yves Félix Montagne, maître de conférences, Unité de recherche « Édition, Littératures, Langages, Informatique, Arts, Didactique, Discours 》 (ELLIAD), Université de Franche-Comté. Denis Loizon, maître de conférences habilité à diriger des recherches, Institut de recherche sur l'éducation (IREDU), Université de Bourgogne.

${ }^{2}$ Évaluation de la politique publique sur la formation continue des professeurs du premier degré, Rapport n²017-035, Juin 2017.

${ }^{3}$ Pseudonyme choisi par le sujet lors du premier entretien.
} 
Le postulat de l'existence de l'inconscient amène à considérer le concept de sujet divisé. Le sujet de la psychanalyse n'est ni le « Je » de Descartes, ni l'individu de la psychologie, ni l'acteur de la sociologie. Divisé entre conscient et inconscient, besoin et désir, plaisir et satisfaction, pensé et dit, le sujet est scindé par ses structures subjectives.

Freud (1923) structure le psychisme à travers trois instances - le Ça, le Moi et le Surmoi - dont les relations déterminent la vie psychique du sujet. Le Ça renvoie à l'instance pulsionnelle exprimée sans retenue morale ou sociale. Le Moi correspond à l'instance de la représentation, c'està-dire la façon imaginaire dont le sujet se représente dans la réalité sociale. Le Surmoi est l'instance psychique responsable de l'intériorisation des devoirs et interdits fondamentaux.

À la suite de Freud, Lacan envisage le concept de sujet en tant qu'effet de signification des actes et du discours d'un être humain parlant. Cette définition du sujet, distincte du Moi freudien, s'adosse au cadre théorique proposé par Lacan (1963), organisé autour de l'articulation entre les concepts de Réel, Symbolique et Imaginaire.

L'Imaginaire renvoie aux images, aux représentations, aux identifications. Le Symbolique correspond à l'instance psychique responsable du langage et des effets d'agencement de relation entre les mots qu'émet et reçoit un sujet dans ses liens avec les autres. Le Réel est l'impossible (Lacan, 1963) à décrire ni par des mots ni par des images. II désigne ce qui est sans que l'on puisse le prévoir ou l'éviter et qui reste définitivement en soi. II devient alors la limite du savoir.

En considérant que « les êtres humains ne sont pas que des êtres de raison mais aussi des êtres parlants et passionnels ", il est possible d'envisager que "cela les divise dans leur conduite corporelle et dans leur pensée » (Labridy, 2006, p.6). La prise en compte du cadre théorique de la psychanalyse offre l'opportunité d'un éclairage singulier sur les causes des résistances d'un sujet à réinvestir les contenus dispensés en formation continue et donc d'éclairer quelque chose de l'expression de son désir.

Dans sa définition usuelle : " aspirer à avoir » et dans son acception étymologique : desiderare (regretter l'absence de l'astre), le désir implique une attente qui doit être satisfaite. II y a dans le désir la marque d'un manque mais en même temps la dimension d'un projet et/ou d'une recherche.

Le désir correspond dans la théorie freudienne à la satisfaction de son accomplissement mais aussi au souvenir d'une expérience de satisfaction passée. Le désir de la psychanalyse est inconscient. Différencié du besoin il reste souvent imperceptible par le sujet « désirant » bien qu'organisant en permanence ses actes et ses paroles.

Dans cette recherche, le non-réinvestissement des contenus de formation ne serait-il pas l'expression de quelque chose du désir de Gilles ? Ses résistances ne révèlent-elles pas les écarts entre ses besoins de formation (dans la réalité de ses cours) et sa demande (dans le Réel pulsionnel de son être) traduisant en cela le rapport du sujet à son désir. Et donc une impossibilité à faire usage de ces contenus dans l'exercice de son métier?

Ce que Lacan a transmis de l'impossible est la concrétisation de la non-satisfaction pleine et entière de son désir. De cet «impossible », Lacan (1953) en fait un symptôme, en tant que formation inconsciente témoignant d'un conflit psychique. L'impossible correspond à l'instance psychique du Réel. Le Réel renvoie ici à l'idée d'une formalisation impossible, "ce qui insiste à ne pas s'inscrire " (Cathelineau, 2001). Le non-réinvestissement des contenus de formation ne serait-il pas une part de l'impossible de Gilles, faisant en cela écho à un savoir « pas tout transmissible » (Terrisse, 1994) dans la situation d'enseignement.

Le Réel qui surgit dans « l'épreuve » de l'enseignement est un « impossible à supporter » (Terrisse, 2009). L'impossible à supporter est à différencier de l'impossible. "Supporter » évoque la 
dimension de la charge, de la souffrance. Le Réel est l'impossible à supporter car il se présente comme une urgence, un débordement du corps et de la pensée.

La didactique clinique (Carnus \& Terrisse, 2013) fait de cet «impossible à supporter » un outil conceptuel pour analyser les manifestations (en paroles et/ou en actes) d'un sujet confronté à « l'épreuve » de l'enseignement. Ainsi, le réinvestissement des contenus de formation en situation d'enseignement serait-il « insupportable » à Gilles, pour des raisons singulières, pas toujours conscientes, de surcroît quand il s'agit de natation?

\section{- La natation comme support d'enseignement}

La natation, dans le contexte du premier degré, peut s'envisager en référence au Savoir Nager, que Patrick Pelayo (1999) définit comme le « fait d'évoluer dans un milieu aquatique appréhendé dans son volume sans utilisation d'équipement particulier et sans avoir la possibilité immédiate de reprise d'appui ". Cette aisance aquatique nécessite la construction d'une motricité organisée autour des fonctions d'équilibration, de propulsion, de respiration et de prise d'information (GalPetitfaux, 1993). La transformation de ces fonctions pour passer du «terrien au nageur » (Catteau, 2008) caractérise le progrès moteur aquatique.

Ces nouvelles modalités de mobilisation du corps en natation appellent le sujet à se confronter à des bouleversements au niveau de la perception de son corps. Pour certains sujets il s'agirait d'une « perte de l'objet corporel représentable » (Yahyaoui, 2006), associée à une perte de maîtrise. La confrontation à l'eau crée une remise en cause du référentiel de terrien qui peut s'envisager aussi du côté fantasmatique et imaginaire. Marie-Hélène Delanöe-Brousse (1978) pour les interrelations, Marc Lévèque (1985) pour les sensations, ont montré en quoi les logiques internes et les problèmes fondamentaux des activités physiques sportives et artistiques, étaient des terreaux d'accueil et d'expression pour l'inconscient d'un sujet.

À la perte de maitrise du corps du sujet dans le milieu aquatique, viendrait s'ajouter, dans l'imaginaire enseignant, la perte de maitrise de l'enseignement dans ce milieu auquel sont associées les modalités pédagogiques transmises en formation.

L'enseignement de la natation, dans sa relation au milieu aquatique, ferait surgir l'intime du sujet comme étranger, inconnu au point d'être angoissant. Cette "inquiétante étrangeté 》 (Freud, 1919) viendrait, dans ce cas, faire obstacle au réinvestissement des savoirs de la formation. Dès lors, les contenus d'enseignement proposés en formation ne pourraient-ils pas faire écho à des phénomènes angoissants chez les professeurs des écoles ?

L'environnement inhabituel de l'enseignement de la natation place également les professeurs des écoles face à la confrontation au regard de l'autre. Le partage de l'espace de pratique notamment avec les maitres-nageurs, " ex-pose » le savoir de l'enseignant. Dans ce jeu de perceptions et de ressentis (voir, se faire voir, être vu), l'enseignant a une place à tenir qui n'est pas sans lien avec un idéal de maîtrise à enjeu narcissique (Pujade-Renaud, 1983).

L'enseignement de la natation, à travers la prise en compte de la dimension symbolique de l'eau, du rapport singulier de chacun à cette pratique et du contexte d'enseignement spécifique, semblerait favoriser la manifestation de l'inconscient du sujet. Se centrer sur les tensions vécues par le sujet dans l'épreuve d'une formation " natation » permettrait de mieux comprendre certains phénomènes du processus d'enseignement qui échappent au cadre didactique «traditionnel ». En conséquence, le rapport de certains professeurs des écoles aux contenus de la formation pourrait traduire l'expression d'un écart entre leur appropriation et leur réinvestissement.

\section{- La formation continue comme modalité de formation}

La formation support à cette étude s'inscrit dans le cadre d'une politique institutionnelle en faveur de l'enseignement de la natation dont l'objectif est de permettre le progrès moteur aquatique et la construction de l'autonomie des élèves. 
Ce double objectif permet de distinguer deux types de savoirs professionnels à développer en référence à ceux identifiés par Marguerite Altet (1996) :

- des « savoirs à enseigner » : il s'agit ici de savoirs relatifs à la construction d'une motricité aquatique chez les élèves. Les contenus de la formation sont axés, par exemple, sur la connaissance des transformations motrices liées au milieu aquatique, sur la capacité à élaborer des situations d'apprentissage et à réguler les conduites motrices des élèves ;

- des "savoirs pour enseigner", autrement dit des savoirs procéduraux sur les mises en œuvre pédagogiques. Ces contenus structurés autour du «comment faire apprendre » portent sur les éléments d'une démarche enseignement apprentissage visant l'autonomie chez les élèves. Ils s'illustrent par la connaissance, la conception et la régulation de dispositifs sous forme d'ateliers favorisant la quantité d'action, l'autoévaluation et la mise en projet des élèves.

L'ensemble de ces contenus s'opérationnalise selon une diversité de modalités en alternant des temps de pratique physique, de pratique pédagogique, d'observation de séance et d'analyse de comportements moteurs.

Par l'articulation de "savoirs sur la pratique, par la pratique et pour la pratique » (Terrisse, 2000), la formation propose des contenus favorisant un "savoir enseigner la natation ». Aussi, quelles raisons pousseraient des professeurs des écoles à ne pas réinvestir ces contenus ?

Les travaux de Thierry Fleitz (2004) montrent chez les enseignants une utilisation différentielle des contenus de formation en éducation physique et sportive. Au travers de l'identification d'un lien ternaire entre l'enseignant, le savoir et son utilisation, il dégage que la dimension "opérative » d'un contenu de formation serait liée au sujet. Aussi, ne pourrait-on pas penser, à l'inverse, que la dimension «non opérative » des contenus de formation serait révélatrice du rapport au savoir du sujet?

Envisagé du côté du sujet psychique, le rapport au savoir est entendu comme un rapport du sujet à son propre désir, désir de savoir. "Mise en lien qui ne norme pas le savoir mais une liaison d'un sujet et d'un objet » (Beillerot, 1989, p.67), le rapport au savoir est à comprendre comme la valeur affective qu'un sujet accorde au savoir, au fait d'apprendre. Cette valeur subjective et affectante renvoie à quelque chose de la relation d'objet telle qu'identifiée par Freud (1917), et précisée par Lacan (1956), le sujet considérant le savoir comme un objet oral à ingurgiter, ou à régurgiter d'un point de vue fantasmatique.

Cela signifie que les actes d'enseignement et d'apprentissage ne peuvent se concevoir uniquement du point de vue d'une rationalité externe, qui exclurait les sujets, leur histoire de vie, leurs rapports intimes à l'activité transmise en formation.

Le cadre théorique de la psychanalyse permet de questionner ce travail à partir de la prise en compte de la dimension de l'inconscient. Autrement dit, dans l'activité d'enseignement de Gilles, certaines choses se feraient à « l'insu de son plein gré ».

Aussi, la question qui émerge serait de savoir si le non-réinvestissement des contenus transmis en formation traduirait un rapport au savoir révélateur de la division du sujet.

\section{Cadre méthodologique}

\section{- La clinique d'orientation psychanalytique}

Basée sur «l'analyse interprétative de cas individuels » (Revault d'Allonnes, 1989), la clinique analytique vise à produire des savoirs sur un phénomène à partir du récit des sujets entendus et acceptés dans leur singularité. Le chercheur s'intéresse à la parole du sujet qu'il rencontre et écoute à propos d'une expérience ciblée. Dans cette recherche, le cadre clinique permettra d'étudier la singularité de Gilles dans la façon dont son rapport au savoir s'expose quand il parle. 
L'orientation psychanalytique considère que les mots ont un effet opératoire qui dépasse l'effet syntaxique : un effet subjectif. La parole produite sera toujours sous-tendue d'affects, pour le locuteur comme pour l'interlocuteur. C'est pour cela que Lacan (1979) a baptisé l'homme «parlêtre », c'est-à-dire un être humain parlant, fondé et divisé par la parole.

"Ce détour par la parole postule que la vérité d'un acte d'enseignement ne peut pas se déduire directement et exclusivement d'une observation » (Montagne, 2013). II y a alors un intérêt à donner la parole à un professeur des écoles quand il agit de façon surprenante. L'analyse de son dire permettra de repérer ce qui se passe à son insu.

Ce cadre méthodologique amène à envisager la position subjective occupée par le chercheur par rapport à son objet de recherche. Elle est à considérer davantage comme un instrument de connaissance que comme un obstacle. Sa prise en compte, en référence à Michel Foucault (1963) vise à pouvoir objectiver la subjectivité de Gilles à l'aide de la subjectivité du chercheur.

\section{- Recueil des données}

Des entretiens non directifs de recherche (Yelnik, 2005) ont servi de méthode de recueil de données. II s'agit d'une technique d'entretien par laquelle le chercheur, au moyen d'une attitude la plus neutre possible, par des questions ouvertes, et des relances fondées par l'équivoque des mots et le poids signifiant qu'ils induisent dans sa subjectivité, cherche à repérer ce qui se dit du sujet dans ce qu'il dit en parlant de lui. Le chercheur demande au sujet de « préciser ce qu'il perçoit de la situation considérée, de ses causes et de son sentiment vis-à-vis de ce que le chercheur a repéré » (Montagne, 2013, p.518).

Dans le cadre du recueil de données, des processus inconscients sont à l'œuvre entre le chercheur et le sujet. Si l'écoute peut être parasitée par les attentes ou les représentations faites sur l'objet, en avoir conscience, si possible, et les analyser " permet de les mettre un peu à distance et d'en limiter les effets négatifs » (Yelnik, 2005, p.136).

Les paroles de Gilles ont été recueillies lors de quatre entretiens de quarante-cinq minutes chacun. Elles ont été enregistrées et retranscrites intégralement.

L'enchaînement des différents entretiens a adopté une logique d'entonnoir. II s'agit, par leur succession, de venir questionner les hypothèses de recherche, en s'appuyant sur le portrait subjectif du sujet dressé lors du premier entretien.

Ces entretiens encadrent l'observation filmée d'une leçon d'EPS de Gilles en natation, empruntant en cela le cadre élaboré par la didactique clinique (Carnus \& Terrisse, 2013). Un des objectifs du deuxième entretien visait à savoir ce que Gilles prévoyait de faire lors de la séance observée. Le troisième entretien a permis, entre autres, d'identifier ce qu'il pouvait dire (ou ne pas dire) sur la séance.

\section{- Analyse des données}

L'analyse et l'interprétation des paroles du sujet sont conduites par le chercheur et engagent sa subjectivité. Pour garantir la recevabilité épistémologique des résultats, leur présentation à d'autres chercheurs, œuvrant dans le champ de la psychanalyse, a permis de valider ou d'infirmer la cohérence théorique et conceptuelle qui existe entre le cas et la lecture qui en sera faite et présentée. Cela participe d'une « vérification externe » (Van der Maren, 1995) en usage dans d'autres champs de recherche en éducation.

L'analyse de la séance filmée porte sur le repérage du réinvestissement ou non des contenus de la formation articulée aux dires de Gilles dans le deuxième entretien (ce qu'il dit qu'il va faire) et dans le troisième (ce qu'il dit qu'il a fait). 
L'analyse des paroles repose sur l'interprétation du discours (Bardin, 2013) qui passe, pour le chercheur, " par le repérage de l'écart entre le contenu latent et le contenu manifeste du discours, par l'écart entre " le dire et le dit » et par la mise en relation des différents temps de la parole de celui qui parle de lui » (Montagne, 2013).

Cette pratique se fonde sur une conception non pas explicative mais interprétative des paroles d'un sujet. II s'agit d'attribuer une signification aux mots contenus dans le discours du sujet, de repérer certaines incohérences et certaines récurrences, afin de faire émerger des manifestations de certaines dimensions de l'inconscient du sujet.

C'est bien par « la mise en rapport entre les mots lus et la subjectivité du chercheur d'une part et les concepts de la psychanalyse d'autre part » (Montagne, 2013, p.521) que quelque chose de la réalité psychique singulière du sujet qui s'exprime va pouvoir être pointé. Pour ce faire, et en adéquation avec la visée exploratoire de cette étude, certains concepts seront définis dans la partie discussion.

\section{- L'étude de cas}

L'étude de cas est une considération des sujets « au cas par cas, au un par un » (Terrisse, 1999, p.66). La démonstration de Bent Flyvbjerg (2006) à propos du cas unique rend recevable au niveau scientifique de s'intéresser à un seul sujet pour construire du savoir. En effet, le sujet n'est pas considéré pour lui-même, mais pour comprendre en quoi sa structuration inconsciente lui permet de vivre d'une certaine façon une situation de vie particulière mais néanmoins traversable par l'ensemble des êtres humains.

L'impossibilité de Gilles à enseigner la natation proposée renseigne en cela d'autres positions subjectives résistantes, dans d'autres domaines des activités humaines. S'il n'y a pas d'invariant de posture ou de structure psychique de l'embarras de la natation dans le cas de Gilles, il se repère le lien d'un sujet à quelque chose qui le bloque dans une réalisation sociale. Cela peut donc renseigner la nature et les causes de résistances d'enseignants vis-à-vis d'autres disciplines, ou de professionnels dans d'autres champs sociaux.

Professeur des écoles, Gilles enseigne à des classes de $\mathrm{CP}^{4}$ depuis une quinzaine d'années. $\mathrm{Ce}$ niveau de classe est concerné par l'enseignement de la natation dans la ville où il exerce. Le choix de recueillir la parole de Gilles est lié à plusieurs éléments. II enseigne la natation depuis de nombreuses années, sa classe bénéficie de cet enseignement et il a participé à une formation relative à la natation.

\section{Résultats : mise en évidence du symptôme}

Les résultats mettent en évidence ce qui fait symptôme dans l'enseignement de la natation chez Gilles, c'est-à-dire les signes visibles de quelque chose de caché qui révèle le nonréinvestissement des contenus reçus en formation. Ils sont issus de l'analyse de l'enseignement de Gilles lors de la séance observée, articulée avec ses dires lors des entretiens.

Un premier résultat montre que Gilles ne réinvestit pas de contenus pédagogiques favorisant la quantité d'action des élèves. Si Gilles précise qu'il prévoit d'en "faire passer un maximum soit par deux soit par trois "5 afin "qu'y restent pas trop longtemps au bord ", l'observation de la situation montre des passages d'élèves de façon individuelle.

Plusieurs hypothèses peuvent être formulées pour caractériser ce décalage entre l'enseignement de Gilles et les propositions de la formation.

\footnotetext{
${ }^{4}$ Cours préparatoire.

${ }^{5}$ Les paroles de Gilles apparaissant en italique et entre guillemets dans le texte.
} 
Ce choix peut être à relier avec le besoin que semble éprouver Gilles à recourir au contact physique : " après quand y passaient en individuel moi je j'les positionnais ». II pourrait également illustrer la modalité de transmission du savoir organisée par la démonstration : "j'ai mis un élève modèle et c'est lui qui qui a guidé le groupe dans le positionnement ». Ces manières de faire se retrouvent dans ses souvenirs d'apprentissage en tant qu'élève où " $y$ a quelqu'un qui montre comment y fallait se mettre... moi j'ai appris comme ça ».

Dans les dires relatifs aux expériences aquatiques de Gilles où " on nous prenait on nous jetait à l'eau enfin on nous jetait c'est un bien grand mot ", l'homophonie repérée entre "mot » et " maux » donne à cette parole une autre signification. La situation d'enseignement où "quand on est à la piscine et pis qu'on est complètement stressé... on a aucune chance d'être à l'aise " montrerait que les choix de Gilles conservent les traces de ses expériences.

Un second résultat pointe que la relation de Gilles aux maitres-nageurs (MNS), en partie liée au partage de l'espace d'enseignement, impacte le rapport à la formation. Cette relation s'illustre dans les interventions et l'observation des MNS.

Gilles semble davantage sensible au savoir du maitre-nageur qu'au savoir du formateur. Lors de la leçon, il propose à ses élèves une situation pour " assurer la continuité du déplacement en se servant d'une posture allongée " présentée et explicitée lors de la formation. L'observation de la séance montre que Gilles ne fait aucune régulation des comportements moteurs de ses élèves. C'est l'intervention d'un MNS qui permet à Gilles a posteriori de réguler le comportement de ses élèves. Ses propos semblent confirmer ce rapport particulier à un savoir disponible immédiatement caractérisé par les MNS «qui savent faire ». Le statut de " maître comme les maîtres tailleurs (...) le maître qui pratique " et la relation de " maître à maître " qu'il attribue aux MNS sont en décalage avec sa perception de la formation où la relation au formateur, de "maitre à élève ", traduit une parole qui "descend mais ne correspond pas à un besoin ". Cet exemple illustre un décalage entre les intentions d'application des savoirs issus de la formation et ceux mobilisés dans la situation réelle. Le rapport de Gilles au savoir le conduit à s'appuyer davantage sur le savoir du MNS que sur celui du formateur.

La relation au MNS apparait également dans le rôle de surveillance de ce dernier qui " est sur le perchoir celui qui est sur... moi ». La perception de ce regard qualifié d' «intrusif » et de " dérangeant " contraste avec la situation de classe où "tu restes quand même libre ". Paradoxalement, l'expression d'un besoin de formation serait d'" observer un collègue volontaire qui ferait une séance de natation ».

La situation d'observation, en exposant le savoir, prendrait une valeur affective différente aux yeux de Gilles en fonction de la place occupée : active (voir) ou passive (être vu). Ces positions ne seraient pas sans effet sur le rapport au savoir en formation.

Le troisième résultat concerne le non-réinvestissement chez Gilles de contenus relatifs à des modalités pédagogiques privilégiant des situations exploratoires où l'élève est invité à analyser et réguler ses conduites motrices.

Lors de la séance, Gilles a recours au contact physique avec les élèves. Lors des entretiens, il le caractérise soit comme une aide (" je tiens juste ton menton et pis j't 'accompagne pour que t'essayes d'aller »), soit comme une manière de faire apprendre (« je j'leur rentre le menton, j'essaye de leur coller le voilà j'leur monte les fesses pour que tout doucement y arrivent à... »). Gilles voit dans cette modalité la possibilité "d'être des fois plus efficace que simplement de dire au gamin ». Cela s'avère être pour lui " le moyen le plus sûr que les élèves heu comprennent c'que je veux ». À aucun moment ne sont présentées en formation des modalités recourant au fait de toucher les élèves pour favoriser leurs apprentissages.

Pour lui « elles se sont faites intuitivement... j'ai pas de souvenirs... je peux pas dire ça c'est un truc auquel j'ai pensé » ; " moi j'ai pas amené de réflexion personnelle là-dessus... enfin person- 
nelle d'enseignant là-dessus ». Sa difficulté à proposer des explications sur les motifs qui l'ont poussé à agir ainsi pointe un acte qu'il ne peut s'empêcher de faire, et dont il ne peut dire davantage.

Ce rapport au toucher se retrouve dans son rapport à la formation où il souhaiterait "pouvoir avoir un stage d'observation dans une classe où il y a beaucoup de manipulations ».

Les dires de Gilles et ses actes d'enseignement en natation font apparaitre dans la relation pédagogique une dimension tactile peu compatible avec les contenus de formation favorisant l'activité exploratoire des élèves.

\section{Discussion}

La structure psychique de Gilles, telle que l'on peut l'interpréter en analysant ses dires, laisse pointer plusieurs obstacles inconscients à la non-réinvestissement des contenus reçus en formation :

- le poids de son déjà-là ne lui permet pas d'utiliser les savoirs transmis ;

- le rapport au savoir de Gilles est dépendant de satisfactions scopique et orale ;

- le désir de toucher les élèves l'éloigne des contenus mettant les élèves en autonomie.

\section{- La natation de la formation, noyée dans le « déjà-là » de Gilles}

La structure psychique de Gilles laisse apparaître plusieurs " déjà-là » issus de son histoire personnelle qui contribuent à structurer son rapport au savoir en agissant comme des " filtres » (Loizon, 2004) à l'utilisation des savoirs de la formation.

Le " déjà-là » est une notion utilisée dans les travaux de didactique clinique (Carnus \& Terrisse, 2009) qui désigne la somme des expériences vécues dans le passé par un sujet, ainsi que ses conceptions et ses intentions, en tant qu'elles influencent de façon non consciente ses actes présents.

Dans la théorie freudienne le " déjà-là » est un concept qui désigne l'effet d'après coup (dans le présent) d'une expérience traumatique et pathogène vécue dans le passé dont le sujet ne se souvient pas mais qu'il ne parvient pas à oublier. II s'agit en fait du retour « dans le Réel d'un point d'imaginaire non symbolisé » (Bessoles, 1995). Le " déjà-là » est la marque sans signifiant d'un évènement qui est dans le sujet (sans qu'il le sache) et qui se manifeste hors du sujet pour colorer son rapport à la réalité et le faire agir (ou l'empêcher d'agir) de façon " in-sensée » face à une situation " banale » de la vie (réinvestir des contenus reçus en formation dans ses propres cours).

Le refus d'une parole "descendante ", " théorique » qui " ne correspond à rien » est également à référer au rapport au savoir inscrit dans son histoire personnelle. II procède d'un processus d'identification à la fonction symbolique du père, en tant que représentation inconsciente idéale. La parole du formateur, en récusant quelque chose de la transmission parentale, l'amène à se rebeller. II le précise en affirmant, à propos des contenus reçus en formation : "ça m'a jamais trop servi ».

Cette demande de savoirs nécessairement pratiques l'amène à ne pas considérer les contenus de la formation, perçus comme descendants et théoriques, et en cela, en entrave l'appropriation.

Sa manière de concevoir l'enseignement de la natation se définit à partir des souvenirs subjectifs de ses expériences de l'apprentissage au cours desquelles il " fallait se débrouiller dans l'eau », et de mécanismes transférentiels sur les méthodes d'apprentissage de ses enseignants qui « montraient ». Ce « déjà-là » participe à l'élaboration d'une démarche d'enseignement organi- 
sée, à la fois, autour d'une approche intuitive, mais aussi suivant un principe de démonstration. La conception subjective de Gilles, issue de son expérience personnelle, fait obstacle au réinvestissement des savoirs de la formation pensés autour d'une rationalité didactique et d'une démarche d'apprentissage privilégiant l'activité exploratoire des élèves.

Le modèle de natation proposé en formation s'oppose au « déjà-là » de Gilles, produit singulier et subjectif notamment de ses expériences personnelles qui agit comme un « filtre " à sa perception des savoirs qui lui sont proposés, empêchant l'incorporation des contenus de la formation et colorisant ce que Pablo Buznic (2013) appelle « la conversion didactique ». C'est-à-dire la conversion du contenu de l'expérience personnelle du sujet (expérience disciplinaire, épistémique et didactique) en contenu d'enseignement élaboré et transmis.

\section{- Le modèle de natation de la formation, lieu d'un « Ça voir » nager insatisfait}

Dans le rapport au savoir qui caractérise Gilles, l'observation revient fréquemment comme condition d'appropriation des savoirs. Ce désir d'observer traduit un rapport à la pulsion scopique qui signifie la satisfaction liée au regard. Ce qui fait dire à Gilles, à propos de l'observation comme modalité de formation : " on en rêve tous d'aller voir comment ça se fait chez les autres ».

Ce fait d'éprouver un plaisir à "voir chez les autres » vient étayer la référence sexuelle de la pulsion scopique où l'individu s'empare de l'autre comme objet de plaisir qu'il soumet à son regard contrôlant. Gilles trouverait dans cette voie active de la pulsion (regarder) une satisfaction.

La pulsion, concept majeur de la psychanalyse, est définie par Freud (1915) comme une poussée psychique qui a sa source dans une zone corporelle, dont le but est de mettre fin à la tension créée d'obtenir un objet satisfaisant pleinement le sujet. Elle trouve son expression sous forme de représentations ou d'affects.

Freud (1920) introduit l'idée qu'il y aurait dans la vie psychique de tout être humain un principe directeur, la pulsion de mort, caractérisé par des conduites paradoxales de destruction et d'autodestruction, bien plus fort que l'aspiration au bonheur ou à la quête du bien, caractérisées par la pulsion de vie. Ainsi des conduites "visibles " d'un être humain, de ne pas réinvestir des contenus reçus en stage natation, pourraient témoigner de son tiraillement entre ces deux forces. $\mathrm{Ce}$ non-réinvestissement serait l'expression de quelque chose de pulsionnel, du côté d'une satisfaction «pas forcément agréable ».

Cette pulsion met également en scène la dialectique regarder, être regardé. Sa forme passive se concrétise lors des temps d'enseignement de natation par l'observation des maitres-nageurs (MNS). Cette observation vient embarrasser Gilles qui ressent ses effets comme "déstabilisant », " dérangeant » et « intrusif », car affectant son idéal du moi d'enseignant.

Ne serait-il pas envisageable de postuler qu'elle agit paradoxalement comme source de plaisir pour Gilles ? En se soumettant au regard contrôlant de l'autre, il en éprouve de la satisfaction. En admettant qu'il n'a "pas de problème avec les gens qui me disent que je [...] c'est pas bien », il procède d'un retournement de l'objet de plaisir sur lui-même.

Ne pourrait-on pas alors penser qu'il existe chez Gilles, dans la situation d'enseignement en natation, une forme de satisfaction masochiste à exhiber son « manque à savoir » qui justifierait ses résistances à l'utilisation de contenus de formation?

Le rapport au savoir de Gilles s'organise du côté d'une pulsion scopique à satisfaire. On pourrait avancer que pour lui, en référence au Ça freudien siège des pulsions, le « Ça voir » fait savoir.

Par ailleurs, le rapport à l'apprendre de Gilles s'organise à travers la pulsion orale qui se traduit par l'idée d'incorporation, représentative de la relation fantasmatique entretenue avec le savoir en tant qu'objet. 
Ses effets se manifestent dans le discours de Gilles à travers le constat d'une demande non satisfaite, ou en tout cas d'une impossibilité à recevoir les contenus, "c'est-à-dire que ça rentre pas ». Cet empêchement à incorporer le savoir de la formation interroge le processus de transmission. En reprenant les voies relatives aux pulsions, on peut penser comme pour la pulsion scopique que Gilles ne se satisfait pas d'être nourri.

La voie passive à la pulsion orale engage à engloutir, digérer avant de pouvoir restituer. Rapporté au savoir, ce processus correspond à la chronologie vécue par Gilles entre le temps de la formation et celui de l'enseignement. II apparaît que Gilles ne s'accorde pas à cette temporalité quand il a besoin pour mener son enseignement de recourir à des fiches pédagogiques car pour lui : «si j'ai pas ça je me perds ».

Au contraire le contexte d'enseignement, in situ, semble offrir à Gilles la possibilité de se faire nourrir dans l'instant par l'intermédiaire de solutions " concrètes ", "pratiques ", " utilisables ». Cette satisfaction de la demande est rendue possible par la présence de MNS. La satisfaction de ce désir, Gilles semble la trouver dans le contexte d'enseignement de la natation, en la présence du MNS qui « réajuste quand y vient ». Cette opposition relative à la façon dont il a besoin de savoir limite l'usage qu'il peut faire des savoirs transmis en formation.

Le rapport au savoir de Gilles se caractériserait du côté de la pulsion orale dans la satisfaction, au-delà de l'atteinte narcissique, à se faire nourrir par les MNS, répondant par des solutions pratiques à un besoin dans l'instant.

\section{- Le modèle de natation de la formation, une entrée dans « l'eau-tre » impossible}

Gilles propose une natation différente de celle de la formation car il y trouve des satisfactions plus accordées avec sa structure psychique. En effet, si les contenus pédagogiques de la formation ne recommandent pas de toucher les élèves, il semblerait à Gilles impossible de les utiliser tant il éprouve de satisfaction à enseigner sa natation impliquant un toucher corporel.

À la lumière de l'analyse de la parole de Gilles, trois causes subjectives peuvent plausiblement expliquer son « impossible » à ne pas recourir à ce contact corporel.

- II ne peut pas ne pas toucher les élèves car au regard de la notion de holding développée par Donald Winnicott (1953), en tenant les élèves, il développe une fonction contenante qui les soutient et leur offre une forme de protection à l'épreuve du milieu aquatique. II signifie l'irréalisable d'un lien non tactile aux élèves par la récurrence dans son discours du signifiant «j'les accompagne ».

- Le désir de toucher les élèves peut aussi s'envisager du côté de l'éros. Ce constat peut être lu comme une forme de satisfaction d'une pulsion sexuelle par le contact corporel avec les élèves. Dans la natation de Gilles pour faire apprendre les élèves, " y a un moment on passe aux... aux actes enfin entre guillemets ».

- L'impossibilité à ne pas toucher les élèves peut aussi s'entendre à travers le statut que Gilles accorde aux élèves. En considérant les élèves comme des objets matériels qu'il « manipule », on peut penser que Gilles s'inscrit dans un processus de déshumanisation mécaniste. II s'arroge, dès lors, la possibilité de recourir au contact physique puisque ses élèves réifiés ne sont plus des sujets mais des objets. Cette piste fait écho à son besoin de manipuler le savoir qui pour lui est « tangible », c'est-à-dire perceptible par le toucher.

Gilles, qui pour "se satisfaire ", doit toucher pour accompagner, toucher pour utiliser, toucher pour déshumaniser, ne peut pas utiliser le modèle de natation de la formation qui le prive du tactile de sa jouissance.

Concept central dans l'enseignement de Lacan (1963a), la jouissance est distincte du plaisir. Lacan situe cette forme de satisfaction humaine du côté de l'impossible à atteindre et du pas toujours agréable (voire du désagréable). Quelque chose en "l'homme recherche et se satisfait du désagréable pour lui » (Montagne, 2015, p.80). 
En appliquant la formule de Lacan (1968, p.43) « ce qui pense, calcule et juge, c'est la jouissance ", il apparait, pour Gilles, que l'impossible à ne pas toucher les élèves dans l'enseignement de la natation pourrait révéler une forme de jouissance chez lui.

Les effets combinés du " déjà-là ", des satisfactions pulsionnelles et de "l'impossible à supporter » renvoyant à un point de jouissance, semblent donc des déterminants subjectifs faisant obstacle à l'utilisation des savoirs de la formation.

Ces déterminants subjectifs rendent compte d'une part d'un " effet sujet » (Montagne, 2013) dans le processus de transmission/appropriation du savoir, illustrant de façon singulière qu'il n'y a pas de savoir sans sujet. II rend compte d'autre part du fait que la natation, activité support, aux savoirs de la formation, par la dimension symbolique qu'elle revêt confronte, ceux et celles qui l'enseignent à l'autre en eux-mêmes, cette « inquiétante étrangeté » (unheimlichkeit) révélée par Freud (1919). La singularité de l'activité natation révèle un effet savoir, autrement dit, il ne peut y avoir de sujet sans rapport à un savoir.

Le rapport entre le sujet et le savoir qui provoque «l'impossible » de Gilles à réinvestir les contenus de la formation est constitutif d'un rapport au savoir singulier et témoigne finalement d'un rapport à son propre désir.

Les résultats de cette étude peuvent faire sien l'avertissement de la psychanalyse qui pose qu'il n'existe pas d'interprétation finale. En effet, "le sens ne s'épuise jamais, il y a toujours un sens suivant possible. L'interprétation de ce qui est dit n'est jamais finie, d'autant qu'aucune énonciation ne se réduit à son énoncé " (Montagne, 2014, p.129). Un autre entretien et la lecture d'un autre chercheur pourraient encore permettre au cas Gilles de se déployer et s'affiner.

\section{Conclusion}

Ce travail se proposait d'analyser et d'interpréter, depuis le champ de la psychanalyse, les effets de l'inconscient à l'œuvre dans le non-réinvestissement des contenus relatifs au savoir enseigner le « savoir nager », issus d'une formation continue.

L'interprétation des dires de Gilles, à travers cet impossible à s'approprier les savoirs de la formation, montre une part de son "rapport au savoir » et de l'orientation que le désir du sujet donne à son usage du savoir.

Cette position engage à repenser l'acte d'intervention d'un formateur d'enseignants et l'évaluation de ses conséquences. Elle nécessiterait de penser autrement la temporalité de la formation continue "natation", en organisant, par exemple, sur la durée d'un cycle d'enseignement des temps de parole et d'analyse de pratique de l'activité d'enseignement. Cette modalité permettrait de développer un repérage pour les participants de leurs propres désirs ou de leurs résistances. La dimension collective de l'analyse de pratique, par la fonction contenante du groupe, faciliterait le passage d'une " posture de praticien à une posture de chercheur sur sa pratique » (Rinaudo, 2015).

En conséquence, il s'agirait pour le formateur d'adopter une posture clinique qui serait conditionnée par la prise en compte :

- de la singularité du sujet issue de son déjà-là ;

- du cas par cas, en considérant l'exemple comme la possibilité d'extraire une hypothèse sur le général ;

- de la vérité subjective du sujet, en acceptant de « dire que oui » (Lacadée, 2007) à la vérité subjective du sujet même si elle dénote avec les propositions de formation et ensuite trouver une façon de faire avec ; 
- de son implication et sa part de responsabilité vis-à-vis de ce qu'il déplore ou ne comprend pas dans les actes des participants à la formation.

Conjointement à l'effet sujet et à l'effet savoir, il serait intéressant d'identifier et d'analyser dans le processus de transmission/appropriation du savoir, un « effet savant » ; autrement dit, l'effet du formateur tel qu'il est subjectivement perçu par ceux qui participent aux formations.

\section{Références}

ALTET Marguerite (1996), "Les dispositifs d'analyse des pratiques pédagogiques en formation d'enseignant : une démarche d'articulation pratique-théorie-pratique ", dans Claudine Blanchard-Laville \& Dominique Fablet, L'analyse des pratiques professionnelles, Paris, L'Harmattan.

BARDIN Laurence (2013), L'analyse de contenu, Paris, Presses universitaires de France.

BEILLEROT Jacky (1989), "Le rapport au savoir : une notion en formation », dans Jacky Beillerot, Alain Bouillet, Claudine Blanchard-Laville \& Nicole Mosconi, Savoir et rapport au savoir : élaborations théoriques et cliniques, Paris, Éditions Universitaires, p.165-202.

BESSOLES Philippe (1995), Le déjà là de la mort et du sexuel, Paris, Theetete Éditions.

BUZNIC-BOURGEACQ Pablo \& TERRISSE André (2013), "Le sujet en position d'enseignant : pratiques et discours de trois professeurs d'EPS débutants avec ou sans expérience personnelle dans l'activité enseignée ", Revue française de pédagogie, ${ }^{\circ} 184$, p.15-28.

CATHELINEAU Pierre-Christophe (2001), Lacan lecteur d'Aristote. Politique, métaphysique, logique, Paris, Association freudienne internationale.

CATTEAU Raymond (2008), La natation de demain : une pédagogie de l'action, Anglet, Atlantica.

CARNUS Marie-France \& TERRISSE André (2013), Didactique clinique de l'EPS. Le sujet enseignant en question, Paris, Éditions E.P.S.

DELANOË-BROUSSE Marie-Hélène (1978), "Les relations affectives dans la pratique sportive ", Dossiers de l'éducateur sportif $2^{\text {nd }}$ degré, Paris, INSEP, p.125-132.

FLEITZ Thierry (2004), "Formation continue et transformation des pratiques enseignantes: le rapport à la formation », Savoirs, n4, p.79-97.

FLYVBJERG Bent (2006), «Five Misunderstandings about Case-Study Research », Qualitative Inquiry, vol.12, n², p.219-245.

FOUCAULT Michel (1963/2015), La naissance de la clinique, Paris, Presses universitaires de France.

FREUD Sigmund

- (1915/2006), Pulsions et destin des pulsions, Paris, Payot.

- (1917/2011), Deuil et Mélancolie, Paris, Payot.

- (1919/1985), L'inquiétante étrangeté et autres essais, Paris, Gallimard.

- (1920/2013), Au-delà du principe de plaisir, Paris, Presses universitaires de France.

- (1923/2010), Le Moi et le Ça, Paris, Payot.

GAL-PETIFAUX Nathalie (1993), Savoir Nager une pédagogie de la natation, Paris, Éditions EPS.

LABRIDY Françoise (2006), « La revue EPS interroge F. Labridy », Revue EPS, n³20, Paris, p.5-12.

LACADÉE Philippe (2007), L'éveil et l'exil, Nantes, Cécile Defaut.

LACAN Jacques

- (1953), La psychanalyse, 1, 1956. Actes du congrès de Rome.

- (1956/1988), Le Séminaire IV, La Relation d'objet, Paris, Seuil.

- (1963/2005), Des-noms-du-père, Paris, Seuil.

- (1963a/2004), Le Séminaire X, L’angoisse, Paris, Seuil.

- (1968/2006), Le Séminaire XVI, d'un autre à l'Autre, Paris, Seuil.

- (1979/2005), Le Séminaire XXIII, Le Sinthome, Paris, Seuil. 
LÉVÈQUE Marc (1985), «Contribution à une lecture différentielle des pratiques sportives », Enfance, n³8/4, p.403-418.

LOIZON Denis (2004), Analyse des pratiques d'enseignement du judo : identification du savoir transmis à travers les variables didactiques utilisées par les enseignants en club et en EPS, Thèse de doctorat, Université Toulouse III.

LOIZON Denis et al. (2005), « La transmission des savoirs : le savoir personnel des enseignants », Revue Savoirs, $n^{\circ} 8$, p.107-123.

MONTAGNE Yves Félix (2013), " "Savoir-y-faire" avec les élèves; repérer une compétence professorale d'énonciation par une étude de cas ", Revue des sciences de l'éducation, n³7, p.507-541.

MONTAGNE Yves Félix (2014), “ Les enseignants face aux élèves en difficulté scolaire : l'intérêt des Groupes de Parole et d'Analyse de Pratique (GPAP) », Les Sciences de l'éducation - Pour l'Ére nouvelle, n47(1), p.117-144.

MONTAGNE Yves Félix (2015), «Le SK-8, comme symptôme d'un adolescent: regard psychanalytique et perspectives en EPS », Staps, n¹08(2), p.73-83.

PELAYO Patrick, MAILLARD Dominique, CHOLLET Didier \& ROZIER Denis (1999), Natation au Collège et au Lycée, Paris, Éditions EPS.

PUJADE-RENAUD Claude (1983), Le corps enseignant dans la classe, Paris, ESF.

REVAULT D'ALLONNES Claude (1989), La démarche clinique en sciences humaines, Paris, Dunod.

RINAUDO Jean-Luc (2015), «Analyse clinique des pratiques en formation et professionnalisation », dans Des professionnalités sous tension : quelles (re)constructions dans les métiers de l'humain ?, Louvain-la-Neuve, De Boeck Supérieur, p.109-120.

TERRISSE André (1994), La question du savoir dans la didactique des APS : essai de formalisation, Note de synthèse pour l'habilitation à diriger des recherches (HDR), Université Toulouse III.

TERRISSE André (1999), « La question du rapport au savoir dans le processus d'enseignement-apprentissage : le point de vue de la clinique », Carrefour de l'éducation, n7, p.62-87.

TERRISSE André (2000), “Épistémologie de la recherche clinique en sports de combat », dans André Terrisse, Recherches en Sports de combat et Arts Martiaux. État des lieux, Paris, Éditions Revue EPS, p.95-108.

TERRISSE André \& CARNUS Marie-France (2009), Didactique clinique de l'éducation physique et sportive (EPS). Quels enjeux de savoirs ?, Paris, De Boeck.

VAN DER MAREN Jean-Marie (1995), Méthodes de recherche pour l'éducation, Bruxelles, De Boeck.

WINNICOTT Donald (1953/2008), La mère suffisamment bonne, Paris, Payot.

YAHYAOUI Yasmina (2006), « L'éthique peut-elle se transmettre ? », Revue des cahiers pédagogiques, n441, p.26-28.

YELNIK Catherine (2005), «L'entretien clinique de recherche en sciences de l'éducation », Revue Recherche et Formation, n50, p.133-146. 